\title{
Ginsenoside Rh2 and Rg3 inhibit cell proliferation and induce apoptosis by increasing mitochondrial reactive oxygen species in human leukemia Jurkat cells
}

\author{
TING XIA ${ }^{1-3}$, YING-NAN WANG ${ }^{4}$, CHUAN-XIN ZHOU $^{2}$, LI-MEI WU $^{5}$, YONG LIU $^{5}$, \\ QIAN-HONG ZENG ${ }^{5}$, XIANG-LONG ZHANG ${ }^{1}$, JIA-HUI YAO ${ }^{1}$, MIN WANG $^{1}$ and JIAN-PEI FANG ${ }^{5}$ \\ ${ }^{1}$ Key Laboratory of Industrial Fermentation Microbiology, Ministry of Education, College of Biotechnology, \\ Tianjin University of Science and Technology, Tianjin 300457; ${ }^{2}$ Department of Pediatrics, The Fifth Hospital of \\ Sun Yat-Sen University, Sun Yat-sen University, Zhuhai, Guangdong 519000; ${ }^{3}$ Department of Physiology, \\ Zhuhai Campus, Zunyi Medical University, Zhuhai, Guangdong 519041; ${ }^{4}$ Department of Medical Oncology, \\ Sun Yat-sen University Cancer Center, State Key Laboratory of Oncology in South China, \\ Guangzhou, Guangdong 510060; ${ }^{5}$ Department of Pediatrics, Sun Yat-sen Memorial Hospital, \\ Sun Yat-sen University, Guangzhou, Guangdong 510120, P.R. China
}

Received February 1, 2016; Accepted February 16, 2017

DOI: $10.3892 / \mathrm{mmr} .2017 .6459$

\begin{abstract}
Ginsenoside Rh2 (GRh2) and ginsenoside Rg3 (GRg3) are primary bioactive components in Panax ginseng. The present study aimed to investigate the underlying mechanisms of apoptotic cell-death induced by GRh2 and GRg3 in human leukemia Jurkat cells. The Cell Counting kit-8 assay was used to determine cell proliferation. Apoptosis was detected by nuclear morphologic observation by Hoechst 33342 staining and Annexin V-allophycocyanin and 7-amino-actinomycin D assay. mitoTEMPO, a mitochondrial reactive oxygen species (ROS) scavenger, was used to examine the effects of mitochondrial ROS on cell viability and mitochondrial membrane potential (MMP). Finally, the expression levels of numerous mitochondrial-associated apoptosis proteins were assessed by western blot analysis. These results demonstrated that GRh2 and GRg3 inhibited cell growth and induced apoptosis, and that GRh2 had greater cytotoxicity than GRg3. GRh2 induced generation of more mitochondrial ROS compared with GRg3 in Jurkat cells;
\end{abstract}

Correspondence to: Professor Min Wang, Key Laboratory of Industrial Fermentation Microbiology, Ministry of Education, College of Biotechnology, Tianjin University of Science and Technology, 29, 13th Street Tianjin Economic and Technological Development Area, Tianjin 300457, P.R. China

E-mail: minw@tust.edu.cn

Professor Jian-Pei Fang, Department of Pediatrics, Sun Yat-sen Memorial Hospital, Sun Yat-sen University, 107 Yanjiang Xilu, Guangzhou, Guangdong 510120, P.R. China

E-mail: jpfang2005@163.com

Key words: ginsenoside $\mathrm{Rh} 2$, ginsenoside $\mathrm{Rg} 3$, acute lymphoblastic leukemia, proliferation, apoptosis, reactive oxygen species however, this effect was ameliorated by subsequent treatment with mitoTEMPO. Furthermore, excess mitochondrial ROS induced by GRh2 was more potent than GRg3 in inhibiting cell proliferation and reducing MMP. In addition, expression levels of apoptosis-associated proteins were significantly increased in Jurkat cells treated with GRh2 than GRg3. In conclusion, these findings suggested that GRh2 and GRg3 induce mitochondrial-associated apoptosis by increasing mitochondrial ROS in human leukemia Jurkat cells. GRh2 may more effectively inhibit cell growth and accelerate apoptosis than GRg3. This study provides a potential novel strategy for the treatment of acute lymphoblastic leukemia.

\section{Introduction}

Ginseng, the root of Panax ginseng, has been used worldwide for thousands of years as a herbal drug in oriental traditional medicine (1). Ginsenosides (ginseng saponins), the primary active components of Panax ginseng, have been demonstrated to have anticancer activities, particularly ginsenoside Rh2 (GRh2) and ginsenoside $\operatorname{Rg} 3$ (GRg3) $(2,3)$. GRh2 and GRg3 are protopanaxadiol (PPD)-type ginsenosides, which have one and two glucose moieties at the C3 hydroxyl of PPD, respectively (4). Previously, it has been reported that GRh2 and GRg3 may inhibit growth (5), induce apoptosis (6) and restrict tumor invasion and metastasis $(7,8)$ in mammalian tumor cells.

Acute lymphoblastic leukemia (ALL), the most common type of childhood malignancy, comprises a group of hematologic neoplasms which may be regarded as clonal expansions of B- and T-lymphocytes arrested at an immature stage of differentiation $(9,10)$. T-cell (T) immunophenotypes, associated with poor outcome, have limited prognostic importance in childhood ALL in the context of contemporary treatment $(11,12)$. Therefore, novel anticancer agents are required to further improve survival rates and to avoid serious side 
effects. GRh2 and GRg3 may be novel natural products for ALL therapy. However, the underlying mechanism of GRh2and GRg3-induced cell death in human T-ALL Jurkat cells remains unclear.

Apoptosis is a process of genetically programmed cell death triggered by biological and physical signals, including chemical reagents $(13,14)$. At present, two major signaling pathways exist to induce apoptosis: Intrinsic-mitochondrial and extrinsic-death receptor (15). The mitochondrial pathway involves the regulation of apoptosis by mitochondria and is characterized by the release of mitochondrial intermembrane space proteins (16). Reactive oxygen species (ROS) primarily generate inside mitochondria, and excess ROS results in dissipation of the mitochondrial membrane potential (MMP), leading to the release of cytochrome c and the subsequent engagement of the Apaf-1-pro-caspase-9 apoptosome complex, which activates downstream caspases $(17,18)$. In addition, the B-cell lymphoma 2 (Bcl-2) family of proteins regulate permeabilization of the mitochondrial outer membrane and cytochrome c release (19). $\mathrm{Bcl}-2$ and $\mathrm{Bcl}-2 \mathrm{X}$-associated protein (Bax) have been identified as primary regulators in mitochondrial control during apoptosis (20).

The present study investigated the anticancer properties of GRh2 and GRg3 in Jurkat cells. Cell viability, nuclear morphology and apoptotic levels were examined to evaluate the cytotoxic effects of GRh2 and GRg3. Mitochondrial ROS generation, MMP and mitochondria-associated apoptotic proteins were determined to examine the underlying molecular mechanisms of GRh2- and GRg3-induced cell death in human acute leukemia Jurkat cells.

\section{Materials and methods}

Materials and cell culture. GRh2 and GRg3 were purchased from Beina Chuanglian Biotechnology Institute (Beijing, China). A Cell Counting kit-8 (CCK-8) was obtained from Dojindo Molecular Technologies, Inc. (Kumamoto, Japan). Hoechst 33342 was purchased from Sigma-Aldrich; Merck KGaA (Darmstadt, Germany). Annexin V-allophycocyanin (APC) and 7-amino-actinomycin D (7-AAD) were obtained from BD Pharmingen (San Diego, CA, USA). MitoSOX ${ }^{\mathrm{TM}}$ Red reagent, Roswell Park Memorial Institute (RPMI) 1640 medium and fetal bovine serum (FBS) were purchased from Thermo Fisher Scientific, Inc. (Waltham, MA, USA). MitoTEMPO was obtained from Santa Cruz Biotechnology, Inc. (Dallas, TX, USA). The primary antibodies against cleaved caspase-9 (9501), cleaved caspase-3 (9665), Bcl-2 (4223), Bax (5023), $\beta$-actin (4970) and secondary horseradish peroxidase (HRP)-labeled goat-anti-rabbit antibodies (7074) were purchased from Cell Signaling Technology, Inc. (Danvers, MA, USA).

The human T-ALL cell line (Jurkat cells) was purchased from the Cell Bank of Chinese Academic of Science (Shanghai, China), and cultured in RPMI medium 1640 supplemented with $10 \% \mathrm{FBS}$ at $37^{\circ} \mathrm{C}$ in a $95 \%$ air and $5 \% \mathrm{CO}_{2}$ incubator.

Cell viability assay. Jurkat cells $\left(5 \times 10^{5}\right.$ cells $\left./ \mathrm{ml}\right)$ were plated on a 96-well (100 $\mu 1 /$ well) microplate and treated with 15, 30, 45 or $60 \mu \mathrm{M}$ GRh2 or GRg3. Cell viability was measured by
CCK-8 according to the manufacturer's protocol. Following treatment, $10 \mu \mathrm{l}$ CCK- 8 solution was added, and cells were incubated for $4 \mathrm{~h}$ at $37^{\circ} \mathrm{C}$. The absorbance in each well was measured at a wavelength of $450 \mathrm{~nm}$ using an automated ELISA reader (Tecan Austria GmbH, Salzburg, Austria). $\mathrm{IC}_{50}$ values were calculated using GraphPad Prism software version 5 (GraphPad Software, Inc., La Jolla, CA, USA) from CCK-8 assay data after $24 \mathrm{~h}$.

Nuclear staining with hoechst 33342. Apoptotic nuclei were observed by chromatin staining with Hoechst 33342 . Jurkat cells $\left(5 \times 10^{5}\right.$ cells $\left./ \mathrm{ml}\right)$ were cultured in a 12 -well plate and treated with $35 \mu \mathrm{M}$ GRh 2 or GRg3. After a $24 \mathrm{~h}$ incubation, the cells were washed with PBS three times, fixed with methanol acetic acid for $10 \mathrm{~min}$ and exposed to $1 \mathrm{mg} / \mathrm{ml}$ Hoechst 33342 at room temperature in the dark for $3 \mathrm{~min}$. The nuclear morphology of Jurkat cells was examined under UV illumination with a fluorescence microscope (Olympus Corporation, Tokyo, Japan).

Annexin V/7-AAD flow cytometry assay. Jurkat cells were seeded into 12 -well plates at a density of $5 \times 10^{5} \mathrm{cell} / \mathrm{s} / \mathrm{ml}$ and treated with $35 \mu \mathrm{M}$ GRh2 or GRg3. After a $24 \mathrm{~h}$ incubation, cells were washed twice with PBS and resuspended in $500 \mu \mathrm{l}$ binding buffer (BD Pharmingen). Annexin V-APC and 7-AAD were added away from light for $15 \mathrm{~min}$ at room temperature. The cells were analyzed by flow cytometry (FACScan; BD Biosciences, San Jose, CA, USA) within $1 \mathrm{~h}$. Cells in early apoptosis are Annexin V-APC-positive and 7-AAD-negative.

Measurement of mitochondrial ROS generation. Mitochondrial ROS levels were measured using MitoSOX Red reagent. Jurkat cells $\left(5 \times 10^{5}\right.$ cells $\left./ \mathrm{ml}\right)$ were seeded into 12-well plates and treated with GRh2 or GRg3 in the presence or absence of $50 \mu \mathrm{M}$ mitoTEMPO, a specific mitochondrial ROS inhibitor. Following a $24 \mathrm{~h}$ incubation, cells were collected and stained with MitoSOX Red, and incubated at $37^{\circ} \mathrm{C}$ in the dark for $30 \mathrm{~min}$. MitoSOX Red fluorescence was observed under a fluorescence microscope and measured by a FACScan ${ }^{\mathrm{Tu}}$ flow cytometer.

Measurement of MMP. The JC-1 fluorescent probe (Sigma-Aldrich; Merck KGaA) was used to detect mitochondrial depolarization during the early stages of apoptosis. Jurkat cells received either single or a combination treatment, as previously described, for an incubation period of $24 \mathrm{~h}$. The cells were stained with JC- 1 in the dark for $30 \mathrm{~min}$ at $37^{\circ} \mathrm{C}$ and washed twice with PBS. JC-1 fluorescence was measured by a FACScan flow cytometer within $1 \mathrm{~h}$.

Western blot analysis. Jurkat cells were cultured in 6-well plates and treated with $35 \mu \mathrm{M}$ GRh2 or GRg3 for $24 \mathrm{~h}$. Whole-cell extracts were lysed using radioimmunoprecipitation assay buffer (Sigma-Aldrich; Merck KGaA). The supernatant was collected after centrifugation at $15,000 \mathrm{x}$ g for $15 \mathrm{~min}$ at $4^{\circ} \mathrm{C}$, and heated to $100^{\circ} \mathrm{C}$ for $5 \mathrm{~min}$ and placed briefly on ice. A total of $20 \mu \mathrm{l}$ supernatant was separated by $12 \%$ SDS-PAGE. Following this, protein samples were electrotransferred onto polyvinylidene fluoride membranes. The membranes were blocked with $5 \%$ non-fat dry milk in $1 \mathrm{X}$ PBST buffer $(0.1 \%$ 

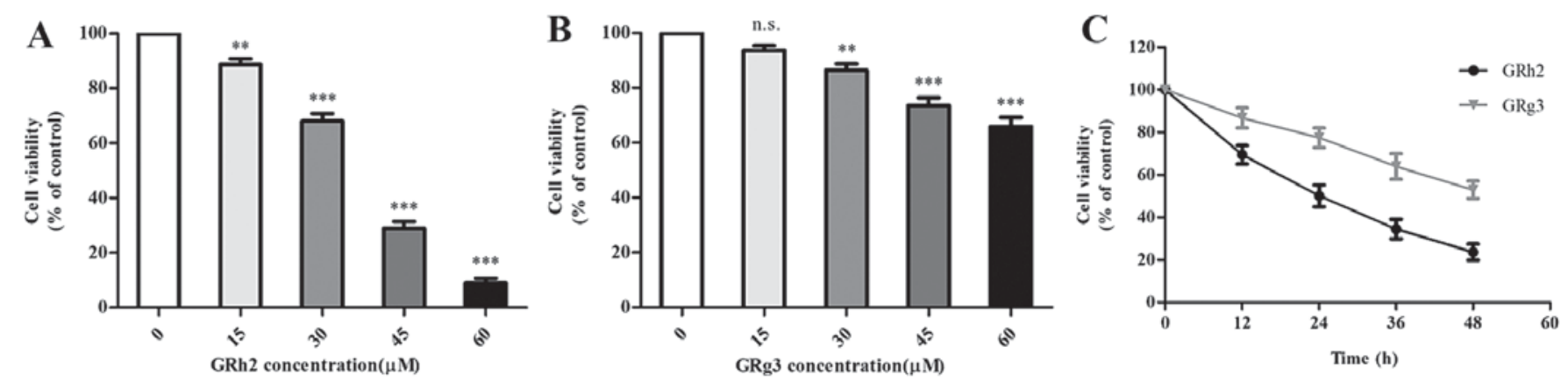

Figure 1. GRh2 and GRg3 treatment inhibits proliferation of Jurkat cells. Jurkat cells were treated with $0,15,30,45$ or $60 \mu \mathrm{M}$ (A) GRh2 and (B) GRg3 for $24 \mathrm{~h}$. (C) Cells were treated with $35 \mu \mathrm{M} \mathrm{GRh} 2$ or GRg3 for $48 \mathrm{~h}$. Cell viability was measured by Cell Counting kit- 8 assay. Data are presented as the mean \pm standard error of the mean. ${ }^{* *} \mathrm{P}<0.01$ vs. $0 \mu \mathrm{M} ;{ }^{* * *} \mathrm{P}<0.001$ vs. $0 \mu \mathrm{M}$. GRh2, ginsenoside Rh2; GRg3, ginsenoside Rg3; n.s., non-significant.

Tween-20 in PBS) for $1 \mathrm{~h}$ at room temperature and incubated in PBST overnight at $4^{\circ} \mathrm{C}$ with the appropriate primary antibody. The membranes were washed with PBST, and incubated in PBST for $1 \mathrm{~h}$ at room temperature with the secondary HRP-conjugated antibody. The immunoreactive bands were visualized by using an ECL kit (32106; Thermo Fisher Scientific, Inc.). $\beta$-actin served as a loading control.

Statistical analysis. All experiments were performed in triplicate and data are expressed as the mean \pm standard error of the mean. Statistical significance was determined by one-way analysis of variance followed by a multiple comparisons test with a Bonferroni adjustment. The analysis was performed using GraphPad Prism software version 5.03. $\mathrm{P}<0.05$ was considered to indicate a statistically significant difference.

\section{Results}

Effect of GRh2 and GRg3 on cell proliferation. The effect of GRh2 and GRg3 on cell viability in human ALL cells was assessed by CCK- 8 assay. Jurkat cells were exposed to 0 , $15,30,45$ or $60 \mu \mathrm{M}$ of GRh2 or GRg3 for $24 \mathrm{~h}$. GRh2 and GRg3 treatment resulted in a dose-dependent decrease in cell viability with $\mathrm{IC}_{50}$ values of $\sim 35 \mu \mathrm{M}$ (Fig. 1A) and $90 \mu \mathrm{M}$ (Fig. 1B), respectively. Jurkat cells were treated with $35 \mu \mathrm{M}$ GRh2 or GRg3 for 12, 24, 36 and $48 \mathrm{~h}$. As presented in Fig. 1C, the survival of Jurkat cells decreased following GRh2 and GRg 3 treatment in a time-dependent manner. Collectively, these results indicated that GRh2 and GRg3 may inhibit proliferation of Jurkat cells, and that GRh2 has a more significant growth-inhibitory effect than GRg3.

GRh2 and GRg3 induce apoptosis in Jurkat cells. To investigate the cytotoxic effect of GRh2 and GRg3, the nuclear morphology of dying cells were examined with Hoechst 33342 staining. As presented in Fig. 2A, following treatment with $35 \mu \mathrm{M}$ GRh2 or GRg3 for $24 \mathrm{~h}$, Jurkat cells exhibited condensed and fragmented nuclei, regarded as a morphological symbol of apoptosis. Nuclear condensation and apoptotic bodies were increased in GRh2-treated cells compared with GRg3-treated cells.

Apoptotic cells induced by GRh2 and GRg3 treatment was assessed using Annexin V-APC and 7-AAD double staining. The results indicated that the population of Annexin V+ and 7-AAD-apoptotic cells was increased in the GRh2- and GRg3-treated groups compared with the control group (Fig. 2B). Additionally, the percentage of early apoptotic cells was $23.23 \pm 3.06 \%$ in the GRh2-treated group and $10.53 \pm 0.98 \%$ in the GRg3-treated group (Fig. 2C). These findings suggested that GRh2 and GRg3 may induce apoptotic cell death in Jurkat cells, and that GRh2 has greater cytotoxicity than GRg3.

Mitochondrial ROS is involved in GRh2- and GRg3-induced cytotoxicity. Previous studies have reported that mitochondria are the major site of ROS production in mammalian cells, but are major targets of detrimental effects $(17,21)$. MitoTEMPO, a specific mitochondrial ROS scavenger, was added to investigate generation of mitochondrial ROS in GRh2- and GRg3-treated Jurkat cells. The results indicated that the red fluorescence intensity was clearly increased in the GRh2- and GRg3-treated groups, and markedly attenuated following concurrent treatment with mitoTEMPO (Fig. 3A). As presented in Fig. 3B, GRh2 and GRg3 significantly increased mitochondrial ROS levels, and mitoTEMPO almost completely blocked GRh2- and GRg3-induced mitochondrial ROS generation. In addition, GRh2 induced generation of more mitochondrial ROS than GRg3 in Jurkat cells. Following this, whether mitochondrial ROS participates in GRh2- and GRg3-induced cytotoxicity in Jurkat cells was investigated. GRh2 was more effective than GRg3 on decreasing cell viability, whereas concurrent treatment with mitoTEMPO markedly attenuated GRh2- and GRg3-induced cell inhibition (Fig. 3C). These results suggested that GRh2 is more potent than GRg3 in inhibiting cell proliferation by mitochondrial ROS generation.

Mitochondrial ROS contributes to dissipation of MMP in GRh2- and GRg3-treated Jurkat cells. To demonstrate the effect of mitochondrial ROS on MMP in GRh2- and GRg3-treated Jurkat cells, MMP levels were examined using a JC-1 sensitive fluorescent probe by flow cytometry. The results revealed that the ratio of JC-1 (red:green) was significantly decreased in cells treated with GRh2 compared with those treated with GRg3. However, concurrent treatment with mitoTEMPO attenuated the loss of MMP in GRh2- and GRg3-treated Jurkat cells (Fig. 4A and B). These results indicated that accumulation of mitochondrial ROS is 
A
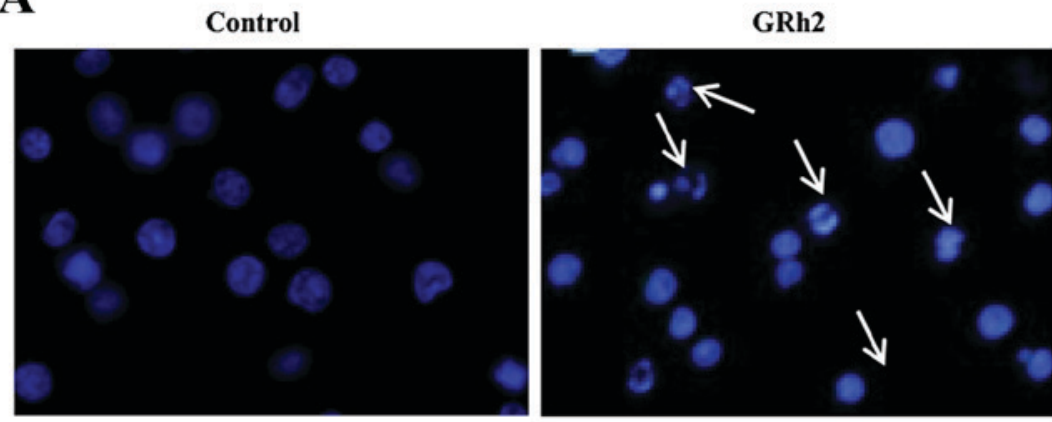

B

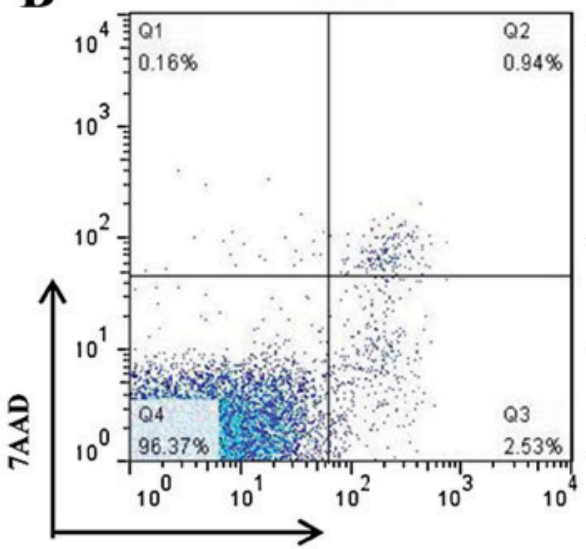

GRh2

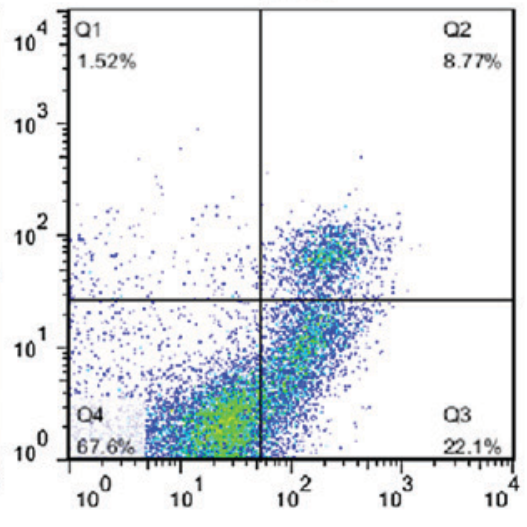

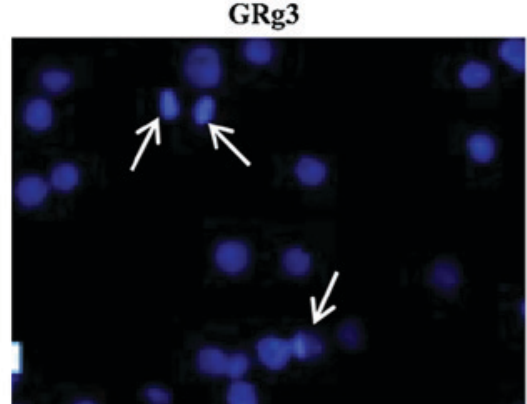

GRg3

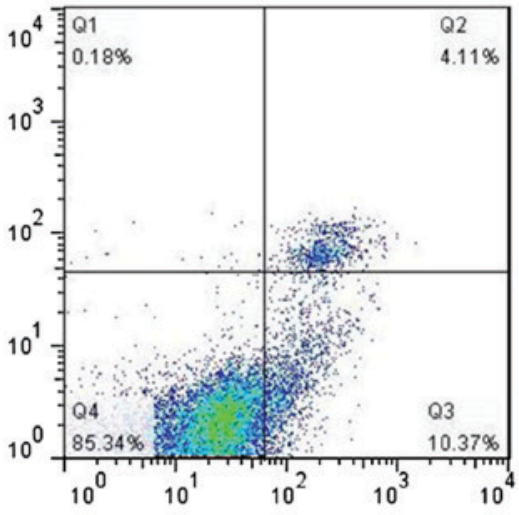

Annexin V-APC

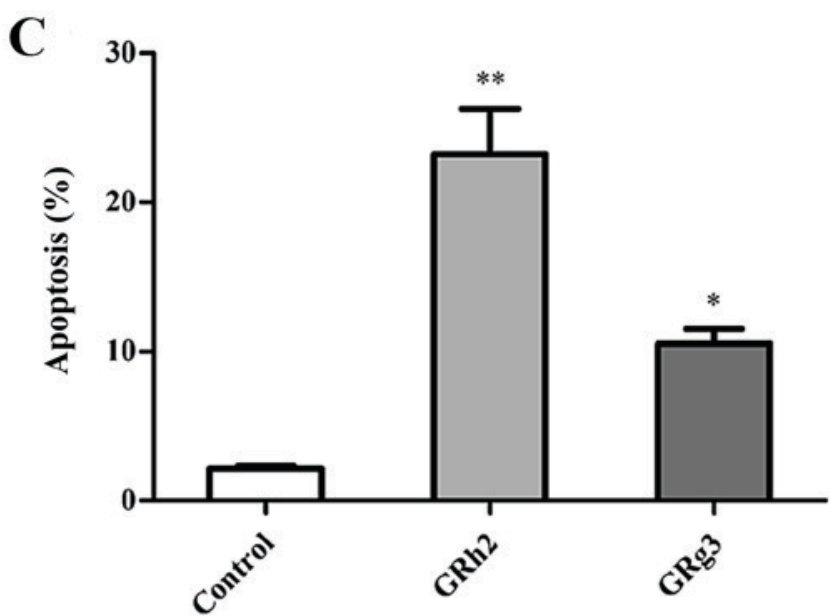

Figure 2. GRh2 and GRg3 treatment induces apoptosis in Jurkat cells. Cells were treated with $35 \mu \mathrm{M}$ GRh2 and GRg3 for 24 h. (A) Apoptotic cell death was examined with Hoechst staining by fluorescence microscopy (magnification, x200). The white arrows indicate morphological alterations in the nuclei of Jurkat cells. (B) Annexin V-APC-positive and 7-AAD-negative stained cells indicate early apoptotic cells. The percentage of early apoptotic cells was analyzed by flow cytometer. (C) Quantification of the percentage of early apoptotic cells. Data are presented as the mean \pm standard error of the mean ( $\mathrm{n}=3 / \mathrm{group}){ }^{*} \mathrm{P}<0.05$ and ${ }^{* * *} \mathrm{P}<0.01$ vs. Control. GRh2, ginsenoside Rh2; GRg3, ginsenoside Rg3; APC, allophycocyanin; 7-AAD, 7-amino-actinomycin D.

more potent than GRg3 in inducing dissipation of MMP in Jurkat cells.

GRh2 and GRg3 induce expression of apoptosis-related proteins in Jurkat cells. To investigate the involvement of the mitochondrial-related pathway in GRh2- and GRg3-induced apoptosis, the expression levels of numerous mitochondrial-associated apoptosis proteins were examined by western blot analysis. It is understood that caspase and Bcl-2 family members serve critical roles in mitochondrial-associated apoptosis (22). The present study demonstrated that protein expression levels of cleaved-caspase-3 and -9 were significantly increased in cells treated with GRh2 compared with cells treated with GRg3 (Fig. 5A and B). As presented in Fig. $5 \mathrm{C}$ and D, the ratio of Bax to $\mathrm{Bcl}-2$ and cytochrome $\mathrm{c}$ was significantly increased in the GRh2-treated group compared with the GRg3-treated group.

\section{Discussion}

ALL is the most prevalent type of childhood malignancy, and T-ALL is associated with poor prognosis (11). To increase survival rates and improve quality of life, novel natural medicines are required to treat T-ALL. GRh2 and GRg3, extracted 


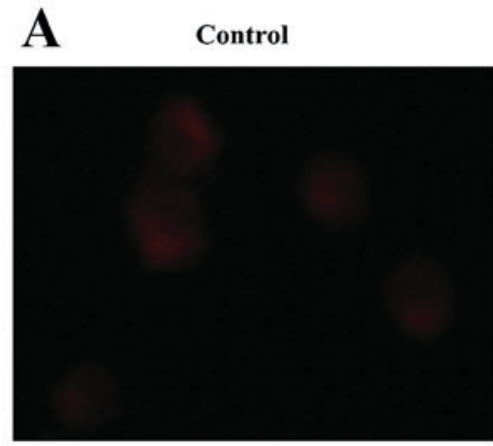

GRh2

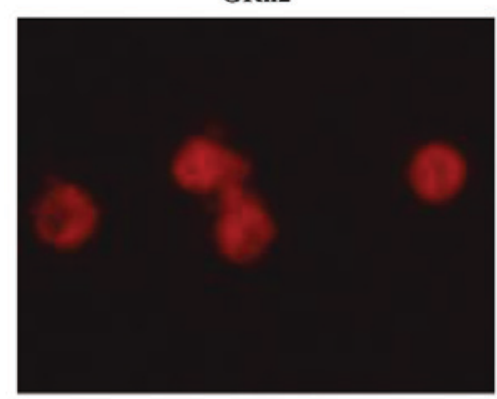

GRg3

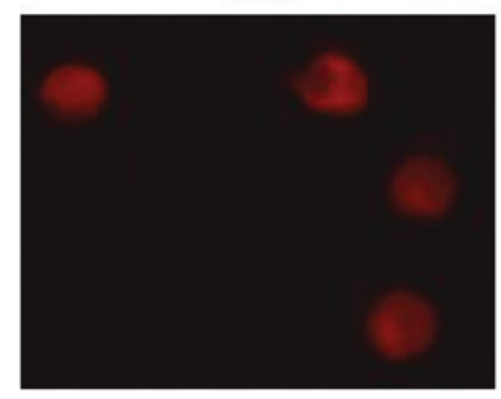

mitoTEMPO

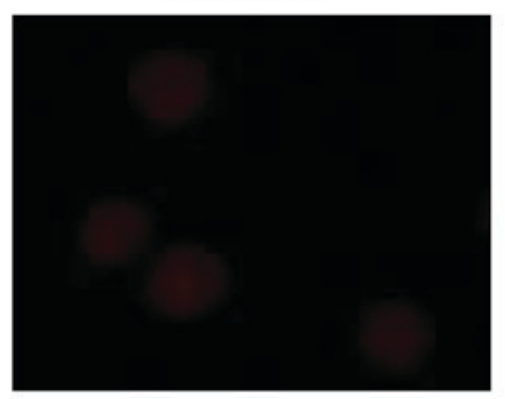

mitoTEMPO+GRh2

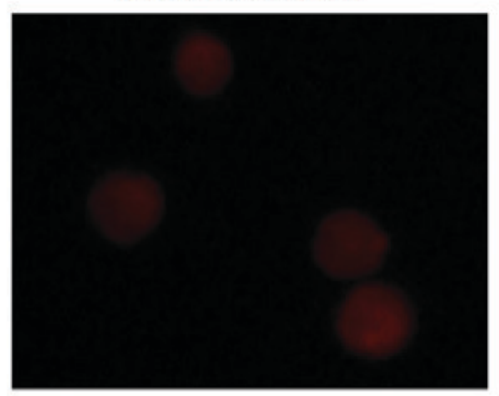

mitoTEMPO+GRg3

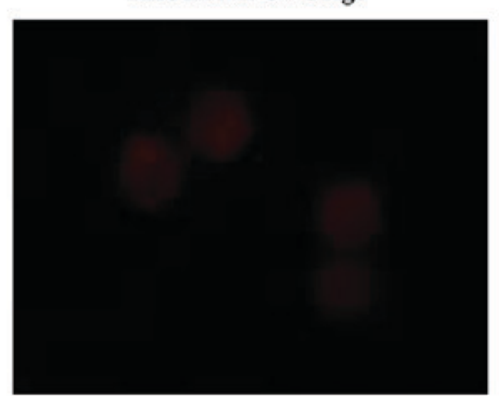

B
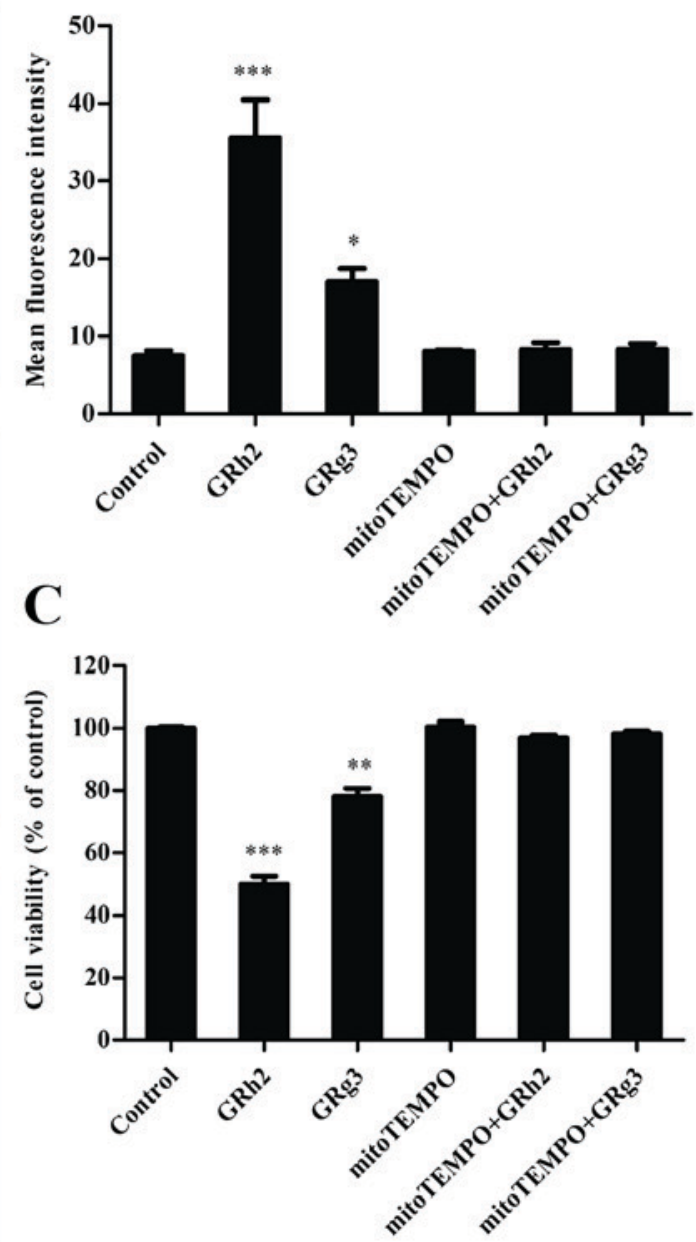

Figure 3. Mitochondrial ROS is involved in GRh2- and GRg3-induced cytotoxicity. Cells were treated with $35 \mu \mathrm{M}$ GRh2 or GRg3 in the presence or absence of mitoTEMPO for $24 \mathrm{~h}$. Mitochondrial ROS levels were measured using a MitoSOX ${ }^{\mathrm{TM}}$ Red reagent. (A) Fluorescence intensity was evaluated by fluorescent microscopy (magnification, x400). (B) Fluorescence intensity was assessed by flow cytometry. (C) Cell viability was measured by Cell Counting kit-8 assay. Data are presented as the mean \pm standard error of the mean $(\mathrm{n}=3 /$ group $) .{ }^{*} \mathrm{P}<0.05,{ }^{* *} \mathrm{P}<0.01,{ }^{* * *} \mathrm{P}<0.001$ vs. Control. ROS, reactive oxygen species; GRh2, ginsenoside Rh2; GRg3, ginsenoside Rg3.

from the root of the Panax ginseng, are recognized as major active anticancer saponins in ginsenosides (23). GRg3 may be metabolized to GRh2 by human fecal microflora (24). It has been reported that GRh2 and GRg3 have anticancer effects on numerous strains of human tumor cells $(6,25,26)$, and GRh2 has a more potent anticancer activity than $\operatorname{GRg} 3(6,27,28)$. The present study investigated the underlying mechanisms of GRh2- and GRg3-induced toxicity, and examined whether mitochondrial ROS contributes to apoptosis via mitochondrial damage in Jurkat cells. These findings provide a potential strategy for T-ALL therapy.

Previous studies have demonstrated that GRh2 and GRg3 are extracted from ginsenosides and have anticancer activities $(5,7,29)$. The present study demonstrated that GRh2 and GRg3 inhibited cell growth in a dose- and time-dependent manner, and GRh2 was significantly more potent at inhibiting Jurkat proliferation than GRg3. Therefore, GRh2 and GRg3 may have apoptosis-inducing activities in Jurkat cells. This hypothesis was supported by the generation of nuclear condensation and apoptotic bodies induced by GRh 2 and GRg3 treatment. Furthermore, GRh2 and GRg3 treatment significantly increased the percentage of apoptotic cells; GRh2 to a greater extent. Collectively, these findings revealed that GRh2 and GRg3 induce apoptosis in the T-ALL cell line, and that GRh2 has greater cytotoxicity than GRg3.

Mitochondria are key regulators of apoptotic cell death. The mitochondrial pathway of apoptosis is activated in response to a number of stress conditions including DNA damage and oxidative stress, which is a common cause of tumor cell death induced by chemotherapeutic agents $(30,31)$. ROS is primarily generated within the mitochondrial electron transport chain during typical cellular metabolism. However, various stimuli, including tumor necrosis factor- $\alpha$, Fas ligand and growth factors, rapidly provoke ROS accumulation in target cells $(32,33)$. In addition, excess ROS induces mitochondrial membrane permeabilization, which, in turn, results in the loss of MMP by activating mitochondrial permeability transition (34). In the present study, mitoTEMPO, a specific mitochondrial ROS inhibitor, was used to assess the role of mitochondrial ROS in GRh2and GRg3-treated Jurkat cells. The results revealed that GRh2 induced increased generation of mitochondrial ROS 

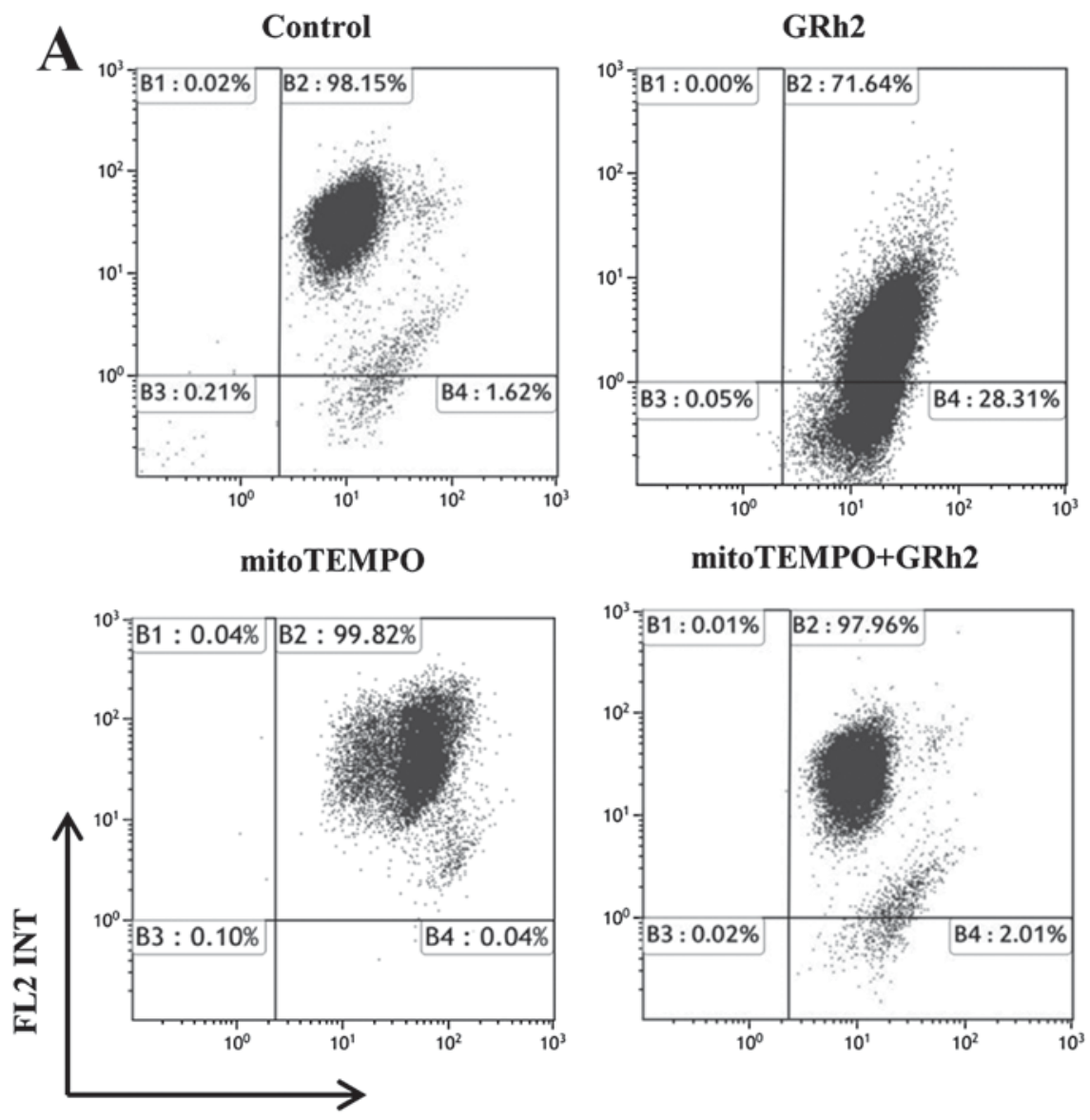

GRh2

mitoTEMPO+GRh2

GRg3

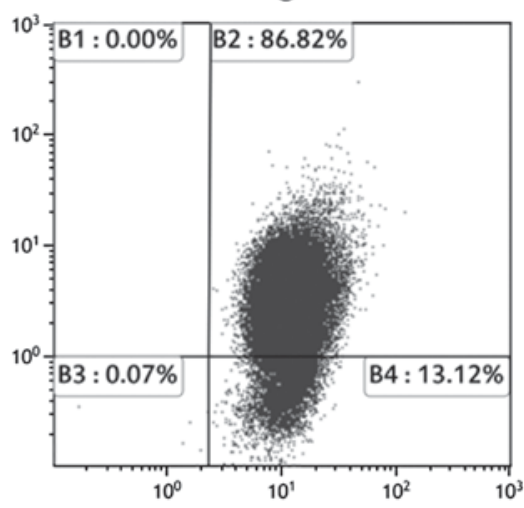

mitoTEMPO+GRg3
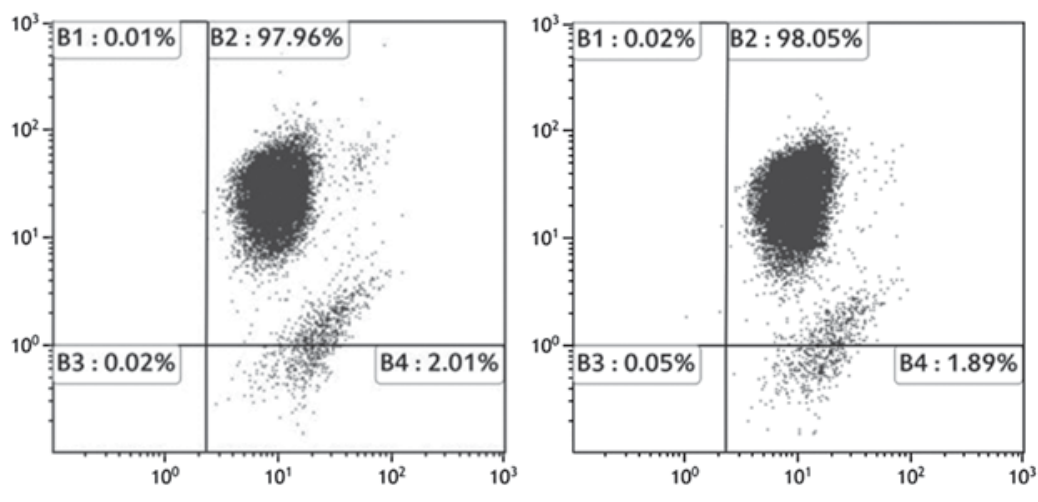

FL1 INT

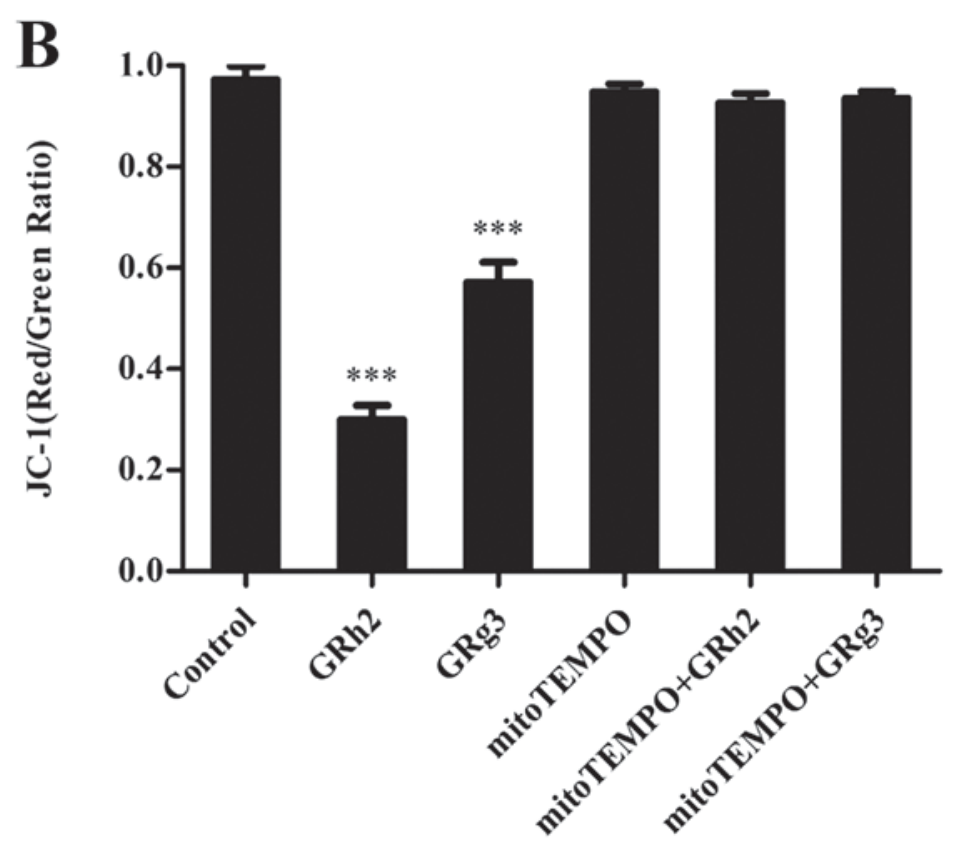

Figure 4. Effect of mitochondrial reactive oxygen species on MMP in GRh2- or GRg3-treated Jurkat cells. Cells were treated with $35 \mu \mathrm{M}$ GRh2 or GRg3 in the presence or absence of mitoTEMPO for $24 \mathrm{~h}$. MMP levels were detected by JC-1 staining. (A) The fluorescence intensity was measured in Jurkat cells by flow cytometry. (B) The ratio of FL2/FL1 reflects MMP level. Data are presented as the mean \pm standard error of the mean ( $\mathrm{n}=3 / \mathrm{group}){ }^{*{ }^{* * *}} \mathrm{P}<0.001 \mathrm{vs}$. control. GRh2, ginsenoside Rh2; GRg3, ginsenoside Rg3; MMP, mitochondrial membrane potential; FL1, fluorescein isothiocyanate signals; FL2, phycoerythrin.

compared with GRg3 in Jurkat cells; however, this effect was ameliorated by subsequent treatment with mitoTEMPO. Furthermore, excess mitochondrial ROS induced by GRh2 was more potent than GRg3 in inhibiting cell proliferation and reducing MMP.
It has been reported that numerous anticancer agents may trigger the release of mitochondrial-associated apoptotic proteins and induce cell death by promoting the intrinsic apoptotic signaling pathway $(18,35)$. It is understood that caspases, another family of kinases, serve an important role in the 

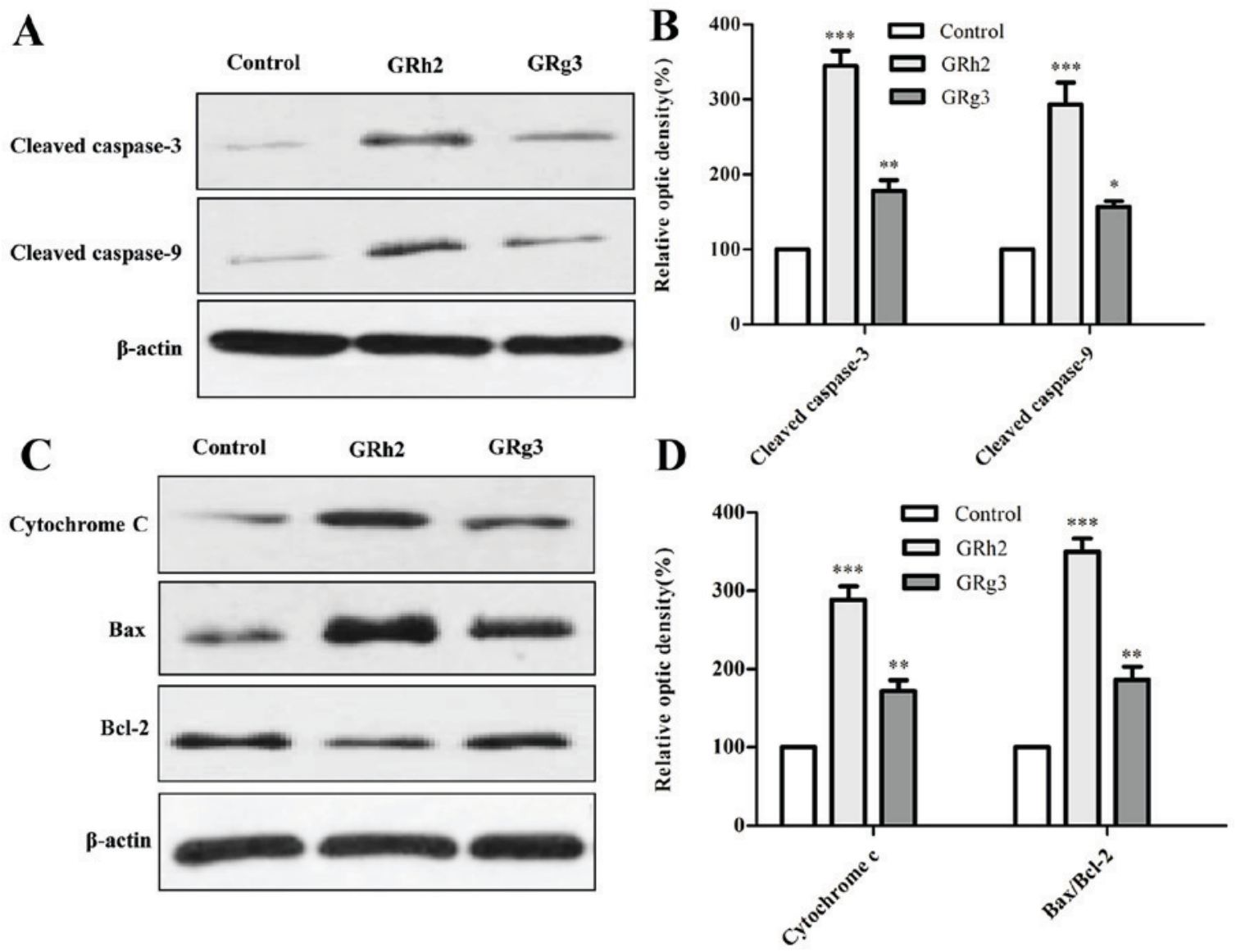

Figure 5. GRh2 and GRg3 treatment induces expression of apoptosis-associated proteins. Jurkat cells were treated with $35 \mu \mathrm{M}$ GRh2 or GRg3 for 24 h. The expression levels of numerous mitochondrial-associated apoptosis proteins were detected by western blot analysis. (A) Representative western blot images and (B) analysis of cleaved caspase-3 and -9 protein expression levels. (C) Representative western blot images and (D) quantification of cytochrome C, Bax and Bcl-2 protein expression levels. $\beta$-actin served as a loading control. Data are presented as the mean \pm standard error of the mean. ${ }^{*} \mathrm{P}<0.05,{ }^{* *} \mathrm{P}<0.01,{ }^{* * * *} \mathrm{P}<0.001$ vs. Control. GRh2, ginsenoside Rh2; GRg3, ginsenoside Rg3; Bcl-2, B cell lymphoma 2; Bax, B cell lymphoma 2-X-associated protein.

regulation of cell apoptosis. Activation of caspase-3 and -9 stimulates mitochondrial cell death signals (36). In addition, gene members of the Bcl-2 family, particularly Bax (an pro-apoptotic gene) and Bcl-2 (an anti-apoptotic gene), are key mediators in regulating the mitochondrial cell death signaling pathway (37). The present study demonstrated that expression levels of apoptosis-associated proteins were significantly increased in Jurkat cells treated with GRh2 compared with GRg3. These findings supported that GRh2 and GRg3 induce apoptosis via mitochondria-dependent signaling pathways, and that GRh2 is more potent than GRg3 in promoting apoptosis of Jurkat cells.

In conclusion, the current study revealed the underlying mechanisms of GRh2- and GRg3-induced cell death in Jurkat cells. GRh2 and GRg3 may inhibit growth and induce apoptosis, and GRh2 has greater cytotoxicity than GRg3. Furthermore, GRh2 inhibits proliferation and induces apoptosis more effectively than GRg3 by stimulating the generation of mitochondrial ROS and promoting the loss of MMP in Jurkat cells. Collectively, these results suggested that GRh2 and GRg3 may be used as potential chemopreventive agents for the treatment of ALL.

\section{Acknowledgements}

The present study was supported by the National Natural Science Foundation of China (grant nos. 81600126,
81570140, 31471722 and 31671851), the National High Technology Research and Development Program of China (grant no. 2013AA102106) and the Program for Changjiang Scholars and Innovative Research Team in University (grant no. IRT15R49) and National Ministry of Science and Technology (grant no. 2016YFD0400505).

\section{References}

1. Attele AS, Wu JA and Yuan CS: Ginseng pharmacology: Multiple constituents and multiple actions. Biochem Pharmacol 58: 1685-1693, 1999.

2. Bak MJ, Jeong WS and Kim KB: Detoxifying effect of fermented black ginseng on $\mathrm{H}_{2} \mathrm{O}_{2}$-induced oxidative stress in HepG2 cells. Int J Mol Med 34: 1516-1522, 2014.

3. Wang JH, Nao JF, Zhang M and He P: 20(s)-ginsenoside Rg3 promotes apoptosis in human ovarian cancer HO-8910 cells through PI3K/Akt and XIAP pathways. Tumour Biol 35: 11985-11994, 2014.

4. Wang P, Wei Y, Fan Y, Liu Q, Wei W, Yang C, Zhang L, Zhao G, Yue J, Yan X and Zhou Z: Production of bioactive ginsenosides $\mathrm{Rh} 2$ and $\operatorname{Rg} 3$ by metabolically engineered yeasts. Metab Eng 29: 97-105, 2015.

5. Kim HS, Lee EH, Ko SR, Choi KJ, Park JH and Im DS: Effects of ginsenosides Rg3 and Rh2 on the proliferation of prostate cancer cells. Arch Pharm Res 27: 429-435, 2004.

6. Park HM, Kim SJ, Kim JS and Kang HS: Reactive oxygen species mediated ginsenoside $\mathrm{Rg} 3$ - and $\mathrm{Rh} 2$-induced apoptosis in hepatoma cells through mitochondrial signaling pathways. Food Chem Toxicol 50: 2736-2741, 2012. 
7. Tang XP, Tang GD, Fang CY, Liang ZH and Zhang LY: Effects of ginsenoside Rh2 on growth and migration of pancreatic cancer cells. World J Gastroenterol 19: 1582-1592, 2013.

8. Kim JW, Jung SY, Kwon YH, Lee JH, Lee YM, Lee BY and Kwon SM: Ginsenoside Rg3 attenuates tumor angiogenesis via inhibiting bioactivities of endothelial progenitor cells. Cancer Biol Ther 13: 504-515, 2012.

9. Richardson RB: Promotional etiology for common childhood acute lymphoblastic leukemia: The infective lymphoid recovery hypothesis. Leuk Res 35: 1425-1431, 2011.

10. Vicente $\mathrm{C}$ and Cools J: The origin of relapse In Pediatric T-Cell acute lymphoblastic leukemia. Haematologica 100: 1373-1375, 2015.

11. Pui $\mathrm{CH}$, Robison LL and Look AT: Acute lymphoblastic leukemia. Lancet 371: 1030-1043, 2008

12. Meleshko AN, Belevtsev MV, Savitskaja TV and Potapnev MP The incidence of T-cell receptor gene rearrangements in childhood B-lineage acute lymphoblastic leukemia is related to immunophenotype and fusion oncogene expression. Leuk Res 30: 795-800, 2006.

13. Reed JC: Apoptosis-regulating proteins as targets for drug discovery. Trends Mol Med 7: 314-319, 2001.

14. Hengartner MO: The biochemistry of apoptosis. Nature 407: 770-776, 2000

15. Ouyang L, Shi Z, Zhao S, Wang FT, Zhou TT, Liu B and Bao JK: Programmed cell death pathways in cancer: A review of apoptosis, autophagy and programmed necrosis. Cell Prolif 45 487-498, 2012

16. Chakraborty JB, Oakley F and Walsh MJ: Mechanisms and biomarkers of apoptosis in liver disease and fibrosis. Int J Hepatol 2012: 648915, 2012.

17. Marchi S, Giorgi C, Suski JM, Agnoletto C, Bononi A, Bonora M De Marchi E, Missiroli S, Patergnani S, Poletti F, et al: Mitochondria-ros crosstalk in the control of cell death and aging. J Signal Transduct 2012: 329635, 2012.

18. Indran IR, Hande MP and Pervaiz S: hTERT overexpression alleviates intracellular ROS production, improves mitochondrial function, and inhibits ROS-mediated apoptosis in cancer cells. Cancer Res 71: 266-276, 2011.

19. Cheng EH, Wei MC, Weiler S, Flavell RA, Mak TW, Lindsten T and Korsmeyer SJ: Bcl-2, Bcl-X(L) sequester BH3 domain-only molecule spreventing BAX-and BAK-mediated mitochondrial apoptosis. Mol Cell 8: 705-711, 2001.

20. Kroemer G: The proto-oncogene Bcl-2 and its role in regulating apoptosis. Nat Med 3: 614-620, 1997.

21. Murphy MP: How mitochondria produce reactive oxygen species. Biochem J 417: 1-13, 2009.

22. Shi Y: Mechanisms of caspase activation and inhibition during apoptosis. Mol Cell 9: 459-470, 2002.

23. Choi JS, Chun KS, Kundu J and Kundu JK: Biochemical basis of cancer chemoprevention and/or chemotherapy with ginsenosides (Review). Int J Mol Med 32: 1227-1238, 2013.
24. Bae EA, Han MJ, Kim EJ and Kim DH: Transformation of ginseng saponins to ginsenoside $\mathrm{Rh} 2$ by acids and human intestinal bacteria and biological activities of their transformants. Arch Pharm Res 27: 61-67, 2004.

25. Li J, Liu T, Zhao L, Chen W, Hou H, Ye Z and Li X: Ginsenoside 20(S) Rg3 inhibits the Warburg effect through STAT3 pathways in ovarian cancer cells. Int J Oncol 46: 775-781, 2015.

26. Guo XX, Guo Q, Li Y, Lee SK, Wei XN and Jin YH: Ginsenoside $\mathrm{Rh} 2$ induces human hepatoma cell apoptosis via bax/bak triggered cytochrome c release and caspase-9/caspase- 8 activation. Int J Mol Sci 13: 15523-15535, 2012.

27. Wang W, Wang H, Rayburn ER, Zhao Y, Hill DL and Zhang R: 20(S)-25-methoxyldammarane-3beta, 12beta, 20-triol, a novel natural product for prostate cancer therapy: Activity in vitro and in vivo and mechanisms of action. Br J Cancer 98: 792-802, 2008.

28. Cheong JH, Kim H, Hong MJ, Yang MH, Kim JW, Yoo H, Yang H, Park JH, Sung SH, Kim HP and Kim J: Stereoisomer-specific anticancer activities of ginsenoside Rg3 and Rh2 in HepG2 Cells: Disparity in cytotoxicity and autophagy-inducing effects due to 20(S)-epimers. Biol Pharm Bull 38: 102-108, 2015.

29. Wu N, Wu GC, Hu R, Li M and Feng H: Ginsenoside Rh2 inhibits glioma cell proliferation by targeting microRNA-128. Acta Pharmacol Sin 32: 345-353, 2011.

30. Favaloro B, Allocati N, Graziano V, Di Ilio C and De Laurenzi V: Role of apoptosis in disease. Aging (Albany NY) 4: 330-349, 2012.

31. Green DR and Kroemer G: Pharmacological manipulation of cell death: Clinical applications in sight? J Clin Invest 115: 2610-2617, 2005

32. Apel K and Hirt H: Reactive oxygen species: Metabolism, oxidative stress, and signal transduction. Annu Rev Plant Biol 55: 373-399, 2004.

33. Fujisawa S, Atsumi T, Ishihara $\mathrm{M}$ and Kadoma Y: Cytotoxicity, ROS-generation activity and radical-scavenging activity of curcumin and related compounds. Anticancer Res 24: 563-569, 2004.

34. Gogvadze V, Norberg E, Orrenius S and Zhivotovsky B: Involvement of $\mathrm{Ca} 2+$ and $\mathrm{ROS}$ in alpha-tocopheryl succinate-induced mitochondrial permeabilization. Int J Cancer 127: 1823-1832, 2010.

35. Yadav N, Kumar S, Marlowe T, Chaudhary AK, Kumar R, Wang J, O'Malley J, Boland PM, Jayanthi S, Kumar TK, et al: Oxidative phosphorylation-dependent regulation of cancer cell apoptosis in response to anticancer agents. Cell Death Dis 6: e1969, 2015.

36. Ola MS, Nawaz M and Ahsan $\mathrm{H}$ : Role of bcl-2 family proteins and caspases in the regulation of apoptosis. Mol Cell Biochem 351: 41-58, 2011.

37. Zhou J, Zhang S, Ong CN and Shen HM: Critical role of pro-apoptotic bcl-2 family members in andrographolide-induced apoptosis in human cancer cells. Biochem Pharmacol 72: 132-144, 2006. 